\title{
Brain abscess and pyocephalus: our experience with treatment
}

\author{
Zdeněk Večeřa ${ }^{1}$, Ondřej Krejčí ${ }^{1}$, Tomáš Krejčí ${ }^{1}$, Jan Krajča ${ }^{2}$, Martin Kanta ${ }^{3}$, Radim Lipina ${ }^{1}$ \\ ${ }^{1}$ Department of Neurosurgery, University Hospital Ostrava, Faculty of Medicine, Ostrava University, Czech Republic \\ ${ }^{2}$ University Hospital Ostrava, Radiodiagnostics Department, Czech Republic \\ ${ }^{3}$ University Hospital Hradec Králové, Department of Neurosurgery, Charles University, \\ Faculty of Medicine in Hradec Králové, Czech Republic
}

\begin{abstract}
Introduction. Pyocephalus always presents serious complications in the treatment of brain abscesses, and is associated with high rates of mortality and morbidity. This study aimed to comprehensively evaluate this understandably feared complication from a purely medical perspective by using an evidence-based approach and drawing comparisons from the available literature, which mostly comprises case reports.

Methods. This was a prospective monocentric study of all patients treated for brain abscesses at the Neurosurgery Clinic of the University Hospital Ostrava between 2012 and 2017. The cohort was divided into two groups for statistical comparison; one group comprised those in which pyocephalus occurred before or during treatment, while the other group comprised patients without this complication. Particular consideration was given to the effect of pyocephalus on morbidity and mortality rates and C-reactive protein levels, as well as to the identification of risk factors, and to its possible therapeutic influence. Patients were followed up for six months.

Results. A total of 43 patients were treated for a brain abscess. An unequivocal diagnosis of pyocephalus was established via CT and MRI brain scans in five cases (11.6\%). In the cohort as a whole, mortality and morbidity rates were $23.3 \%$ and $48.8 \%$ respectively. Among patients with pyocephalus the incidence of mortality and morbidity was $40 \%$ and $66.6 \%$ respectively. The presence of pyocephalus is not a significant predictor of either morbidity ( $p$ 0.575) or mortality ( $p$ 0.664). In patients with pyocephalus, we determined elevated CRP levels on the day of surgery ( $p$ 0.038). The occurrence of epileptic seizures in the acute phase of the disease is associated with a poor outcome ( $p 0.039)$.

Conclusions. Pyocephalus will continue to be a serious complication in the treatment of brain abscesses, although we were unable to determine its utility as a prognostic factor. Patients with this complication have elevated CRP levels on the day of operation.
\end{abstract}

Key words: brain abscess, pyocephalus, mortality, ventriculitis, intraventricular rupture (Neurol Neurochir Pol 2019; 53 (2): 162-168)

\section{Introduction}

A brain abscess is a serious inflammatory illness with an uncertain prognosis. Treatment outcome is influenced not only by a range of underlying factors including age at onset, associated diseases and immune system status, but also by the localisation of the abscess. For example, an abscess close to the ventricle can rupture, leading to the spontaneous effusion of pus into the ventricular system, with the resultant ventriculitis manifesting as sepsis or even septic shock. A diagnosis of pyocephalus can be made initially or as it arises over the course of treatment and is frequently associated with sudden clinical deterioration. The risk of this complication is considered to be higher with the presence of multilocular or deep-seated abscesses close to 
the ventricles [1], and can occur in as many as $35 \%$ of cases $[1,2]$. Mortality rates are high, ranging from $40-85 \%$ [1-4]. In addition to the standard therapies presently carried out, the treatment of pyocephalus can involve ventricular drainage to discharge purulent CSF accompanied by the intraventricular administration of antibiotics, with glycopeptide vancomycin being the one most commonly recommended in case studies [5, 6]. Even so, an established therapeutic strategy for this special subgroup of patients remains controversial, being led as it is by empirical experience of the workplace and each individual patient's clinical development.

\section{Methods}

\section{Study design}

This prospective monocentric study was carried out at the Neurosurgery Department of the University Hospital Ostrava between 2012 and 2017, and comprised all patients who received treatment for a brain abscess during this period. The diagnosis was confirmed upon admission by imaging studies. Initial CT scans were performed on all patients, supplemented by MRI conducted according to the standard protocol consisting of T1- and T2-weighted images, FLAIR, DWI with application of contrast material as standard and also spectroscopy sequencing. For a positive diagnosis of abscess it is always necessary to observe restriction on diffusion-weighted images while taking into account clinical condition and laboratory findings.. Patients with pyocephalus were assigned to one study group, while a second group comprised patients without this complication. Therapeutic algorithms were identical in both groups: initial broad-spectrum empirical antibiotic treatment was modified according to culture findings, preliminary laboratory findings and the establishment of inflammation markers (CRP) while always investigating the origin of the abscess and carrying out surgical treatment. All patients underwent surgery within 24 hours of diagnosis. The type of operation was chosen according to the localisation and the patient's clinical condition. Guided aspiration was carried out on deep-seated lesions in affected areas and residua surgically excised, with samples of the purulent fluid taken away for culture. We carried out external ventricular drainage (EVD) on patients with pyocephalus (excepting that this procedure was not indicated for one patient who was in good clinical condition). Patients were followed up for six months. Outcome was determined on the basis of the modified Rankin Scale (mRS) (Tab. 1). This study was approved by the local Ethics Committee.

\section{Statistical evaluation of the study group}

Statistical comparison was carried out using the IBM SPSS (version 25) statistics program. We focused on morbidity and mortality rates, risk factor identification and potential treatment complications including epilepsy, intraventricular drainage and hydrocephalus.
Table 1. Outcome after six months

$\begin{array}{cc}\text { mRS } & \mathbf{n}=\mathbf{4 3} \\ 0 & 17 \\ 1 & 2 \\ 2 & 0 \\ 3 & 5 \\ 4 & 7 \\ 5 & 2 \\ 6 & 10\end{array}$

\section{Results}

\section{Outcome}

A total of 43 patients ( 24 male, 19 female) were treated, including four children. The average age was 45 years for men and 55 for women. The youngest patient was a 5 year-old boy, the oldest an 86 year-old woman. Pyocephalus was clearly determined by CT and MRI scans in five patients $(11.6 \%$ of cases). Overall mortality in our study reached $23.3 \%$, while the morbidity rate was $48.8 \%$ (mRS 1-5). Among patients with simple abscess, the mortality rate was $21 \%$ and morbidity $36.8 \%$. The causes of death were four cases of accented perifocal oedema accompanied by rapid craniocaudal deterioration, one case of extensive bleeding along the puncture trajectory, two cases of progressive sepsis and one case of cardiopulmonary failure. Among patients with pyocephalus, mortality was $40 \%$ and morbidity $66.6 \%$. In our study, pyocephalus was not a statistically significant predictor of either morbidity ( $\mathrm{p} 0.575$, exact test) or mortality (p 0.664 , exact test). Two patients with pyocephalus died within 14 days with symptoms of persistent sepsis and progressive multiple organ failure. One patient successfully underwent $\mathrm{CPR}$ following spontaneous ventricular fibrillation on the eighth day of treatment. Regarding the morbidity of cured patients, there were two instances of residual hemiparesis and psycho-organicity (mRS 3 ), and one case without any long-term ill effects (mRS 0). Patient characteristics, along with surgical procedures and culture findings, can be found in Table 2.

\section{Seizures}

Five patients (11.6\%) had multiloculated lesions, of which one patient had pyocephalus. The remaining $88.4 \%$ had uniloculated lesions. The total number of lesions was 50 . Most were located at the frontal lobe (38\%, epilepsy risk p 0.309), followed by the temporal lobe (22\%, p 0.778), occipital lobe $(14 \%, \mathrm{p} 0.904)$ and parietal lobe $(12 \%, \mathrm{p} 0.524) .4 / 50$ were at deep-seated structures such as the basal ganglion and brainstem $(8 \%, \mathrm{p} 0.267)$, with $3 / 50$ at the cerebellum (6\%, p 0.224$)$. The effect of abscess location on the incidence of epileptic seizure could not be determined. 
Table 2. Characteristics of patients with pyocephalus

\begin{tabular}{|c|c|c|c|c|c|c|c|c|}
\hline $\begin{array}{l}\text { Pacient with } \\
\text { pyocephalus } \\
\text { (age / sex) }\end{array}$ & $\begin{array}{c}\text { Initial } \\
\text { symptoms }\end{array}$ & $\begin{array}{c}\text { Origin } \\
\text { of infection }\end{array}$ & Localisation & Treatment & EVD & $\begin{array}{l}\text { Culture } \\
\text { findings }\end{array}$ & $\begin{array}{l}\text { Predispos- } \\
\text { ing factors }\end{array}$ & Outcome \\
\hline $44 / M$ & $\begin{array}{l}\text { sopor, neck } \\
\text { stiffness }\end{array}$ & middle ear & $\begin{array}{l}\text { right temporal } \\
\text { lobe }\end{array}$ & excision & yes & $\begin{array}{l}\text { Prevotella sp., } \\
\text { Bacteroides sp. }\end{array}$ & alcoholism & mRS 6 \\
\hline $53 / M$ & fever & unknown & left basal ganglia & aspiration & yes & $\begin{array}{l}\text { Streptococcus } \\
\text { intermedius }\end{array}$ & hypertension & $\mathrm{mRS} 3$ \\
\hline $77 / F$ & $\begin{array}{l}\text { somnolence, } \\
\text { hemiparesis }\end{array}$ & unknown & right parietal lobe & excision & yes & $\begin{array}{l}\text { Streptococcus } \\
\text { intermedius }\end{array}$ & hypertension & $\mathrm{mRS} 6$ \\
\hline $39 / M$ & coma & $\begin{array}{l}\text { dental } \\
\text { infection }\end{array}$ & $\begin{array}{c}\text { right thalamus, } \\
\text { cerebellum, right } \\
\text { P lobe }\end{array}$ & $\begin{array}{c}\text { aspiration } \\
3 x\end{array}$ & yes & $\begin{array}{c}\text { Fusobacterium } \\
\text { nucleatum }\end{array}$ & $\begin{array}{c}\text { alcoholism, } \\
\text { diabetes }\end{array}$ & $\mathrm{mRS} 3$ \\
\hline $5 / M$ & $\begin{array}{l}\text { neck } \\
\text { stiffness, } \\
\text { fever }\end{array}$ & $\begin{array}{l}\text { repeated } \\
\text { respiratory } \\
\text { infection }\end{array}$ & left frontal lobe & aspiration & no & negative & none & mRS 0 \\
\hline
\end{tabular}

The frequency of epileptic seizures, both generalised and partial, was noted over the six-month monitoring period. $32.5 \%$ of patients $(14 / 43)$ were symptomatic in the acute phase of the illness. Ten patients exhibited partial seizures, with generalised seizures in the remaining four, while 11 cases exhibited one-off incidences in the initial or early stage of the illness up to 72 hours post diagnosis, with no recurrence following treatment. In one case it was necessary to place the patient in a barbiturate-induced coma after suffering status epilepticus. This patient died shortly thereafter from cardiorespiratory complications. The period prescribed for anti-epileptics did not exceed that of antibiotics (a maximum of six weeks). Just one patient required long-term medication for chronic epilepsy after the conclusion of treatment. The occurrence of epileptic seizures in the acute stage of the illness is an indicator of a poor long-term prognosis ( $\mathrm{p} 0.039, \chi^{2}$ test), as are persistent $m R S$ values in the range $2-5$ with various presentations of hemiparesis, and impaired psychological and language function being most typically described. It was not found to affect mortality rates (p 1.000, exact test).

\section{Surgical approach}

Guided aspiration of lesions was carried out in 51.2\% (22/43) of cases. There were six reoperations among this subgroup: three punctures were carried out repeatedly on one patient who had pyocephalus; one operation was extended to include excision after adverse MRI findings postoperatively; one revision of puncture trajectory was performed due to haematoma, and in another case it was necessary to perform hemicraniectomy for accentuated perifocal oedema. Excision was primarily performed in $48.8 \%$ (21/43) of cases. In three cases, reoperation was performed 14 days after the primary operation following ambiguous findings on postoperative MRI studies (i.e. partial persistence of diffusion restriction and no indication of regression in lesion size). In another case, acute revision was necessary due to haematoma in the surgical field with the onset of herniation.
Table 3. Origin of infection

\begin{tabular}{lc} 
Origin of infection & $\mathbf{n = 4 3 ( 1 0 0 \% )}$ \\
\hline unknown & $11(25.6 \%)$ \\
paranasal sinusitis & $6(14 \%)$ \\
otitis media & $4(9 \%)$ \\
haematogenous & $9(21 \%)$ \\
complication of therapy (iatrogenic) & $7(16.3 \%)$ \\
dental & $6(14 \%)$
\end{tabular}

\section{Laboratory findings and origin of infection}

Average initial CRP levels were $26 \mathrm{mg} / \mathrm{l}$ in patients without complications and $82.4 \mathrm{mg} / \mathrm{l}$ in those with pyocephalus. Physiological CRP was present in 19 of our study patients $(44.1 \%)$ on the day of operation $(<8 \mathrm{mg} / \mathrm{l})$. We determined statistically higher CRP levels in patients with pyocephalus (p 0.038, median test).

The underlying cause of the abscess could not be established in a quarter of patients. The most common source was haematogenous spread, followed by infection of the paranasal sinus and teeth. The least common cause was middle ear infection. We noted abscess progression as a postoperative complication in seven patients (Tab. 3). Culture remained sterile in $51.1 \%$ of samples. No mycotic or tuberculous abscesses were treated during this study. Two cases of extended cultivation eventually isolated aerobic Nocardia (Tab. 4). In the pyocephalus subgroup, positive culture was found in $80 \%$ of cases, with one patient in a good clinical state and culture-negative. This was due to intraventricular rupture occurring some time after antibiotic therapy with the patient's condition not indicating drainage. The remaining patients had initial pyocephalus. In these we carried out aspiration to evacuate pus from the affected lateral ventricle. In two patients this was performed as part of the primary operation prior to abscess 

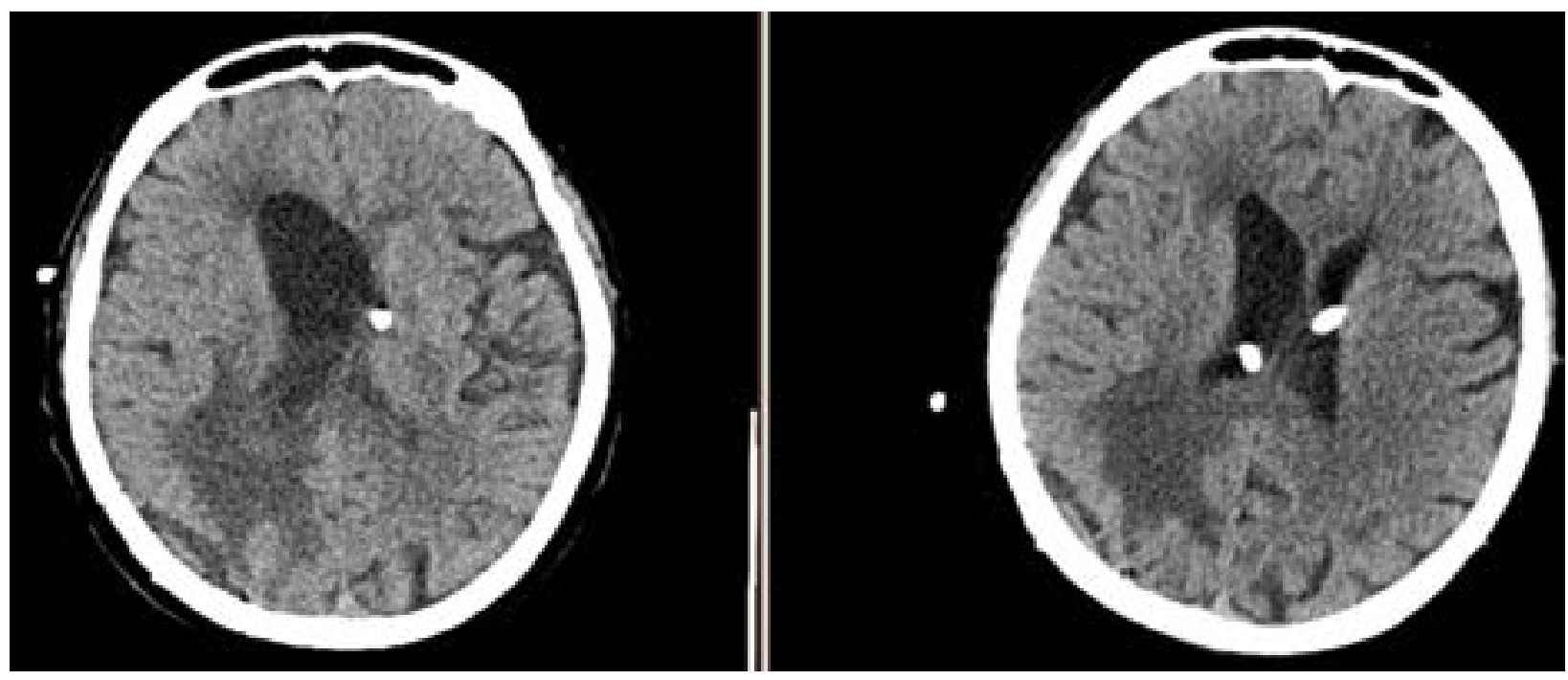

Figure 1. Bilateral external drainage in a patient with isolated lateral ventricles

Table 4. Culture findings

\begin{tabular}{lc} 
Culture findings & $\mathbf{n = 4 3 ( 1 0 0 \% )}$ \\
\hline aerobic origin & $7(16.3 \%)$ \\
anaerobic origin & $6(14 \%)$ \\
mixed cultures & $8(18.6 \%)$ \\
atypical & 0 \\
sterile cases & $22(51.1 \%)$
\end{tabular}

excision. In the remaining two this was performed some time later on the basis of ventricular progression observed by imaging studies. In one case we performed drainage of both ventricles for a bilateral foramen of Monro obstruction (Fig. 1), although this patient died soon after. The remaining patient underwent EVD two days postoperatively for the progression of unilateral hydrocephalus affecting the left lateral ventricle (Fig. 2). In this case, the drain was left in place for four weeks. Repeated attempts to remove were unsuccessful due to the isolated nature of the left lateral ventricle - the situation was resolved by performing guided endoscopic septostomy from the right-hand side. In another surviving patient the drain was left in place for 14 days, after which it was discontinued due to malplacement causing patient discomfort. Regular MRI follow-up found the ventricular system to be stable. VP shunts were not required on any patients in the study. We did not administer antibiotics intraventricularly - all patients were treated intravenously as is standard, initially with the recommended combination antibiotic therapy (2-3 g cefotaxime with 0.5 g metronidazole every eight hours; vancomycin separately in the case of postoperative complications) adjusted as necessary according to culture.

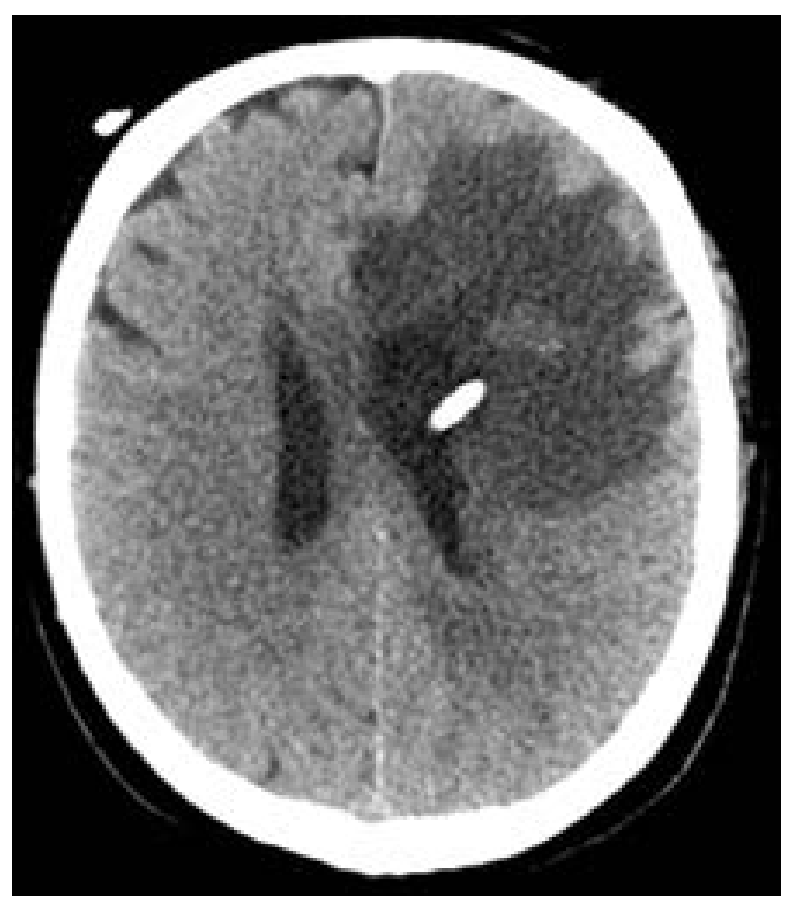

Figure 2. CT image of drained lateral ventricle in a case of unilateral hydrocephalus. Complication was resolved by performing guided endoscopic septostomy

The study included seven immunocompromised patients (16.3\%), none of whom had pyocephalus. In one patient this was due to kidney transplantation, and two others were undergoing radio- and chemotherapy. The remaining four patients were chronically immune-suppressed due to rheumatoid arthritis. Immunosuppression did not affect either morbidity ( $\mathrm{p} 0.240$, exact test) or mortality (p 1.000, exact test). The most commonly-occurring underlying diseases 
were hypertension in 18 patients (36\%) and diabetes in six patients (12\%). Neither hypertension ( $\mathrm{p}$ 0.322) nor diabetes (p 0.526$)$ had any effect on mortality rates.

\section{Discussion}

\section{Outcome and factors related to outcome}

Even with improved imaging techniques and a variety of specific neurosurgical approaches at our disposal, brain abscesses remain a diagnosis with a highly uncertain prognosis that is dependent on their localisation. Overall condition, age and accompanying illnesses all play an important role in treatment outcome.

Our findings concerning mortality and morbidity rates are consistent with current experience. Statistically, the presence of pyocephalus and immuno-suppression did not influence the outcome, nor could we determine that immunosuppression has an influence on the incidence of pyocephalus. The most common underlying conditions were hypertension and diabetes ( $36 \%$ and $12 \%$ respectively). We could not determine if these factors affected the outcome. Xiao et al studied 178 patients treated between 1986 and 2002 and described only four patients with pyocephalus, two of whom died during the study. Neither pyocephalus, diabetes nor cirrhosis were found to effect the outcome. Only initial GCS, immunodeficiency and the presence of underlying disease were found to have a significant influence on the outcome [7]. In the discussion of the range of epidemiological factors involved, we can refer to a retrospective study of brain abscess cases from 2002-2017 conducted by Amornpojnimman et al in Thailand. The authors considered immunosuppression to be the most significant risk factor in the formation of abscesses. Confusion as a symptom of septic encephalopathy is associated with a poor outcome (GOS 1). In this study, there was a high proportion of immunocompromised patients (42\%), although this figure included patients with diabetes (16\%). This study also included 10 cases of HIV (12.3\%), as opposed to none in our study. There was only one case of spontaneous pyocephalus (1.2\%). The most frequently-occurring complication mentioned was hydrocephalus, in $17.3 \%$ of patients, but the study lacked a further appraisal of the treatment or information as to whether EVD was necessary [8]. Kao et al reported that short duration of symptoms, diabetes and cirrhosis were associated with poor outcomes in 53 patients [9]. Another study by the Thai authors concluded that spontaneous intraventricular rupture and fungal abscesses were predictors of a poor outcome [10]. A series of earlier case studies considered pyocephalus as a malign symptom associated with an adverse outcome $[2,3$, 6]. In a study of 179 patients treated between 1986 and 2005, Lee et al identified the risk factors predictive of ventricular rupture to be size and multiloculation of abscesses, and their proximity to the ventricular system. This study included a high overall incidence of intraventricular rupture (34.6\%).
$25.2 \%$ of patients were initially diagnosed and $9.4 \%$ were diagnosed over the course of treatment. Mortality among this subgroup of patients was $26.7 \%$ at three months. The surgical therapy described for this cohort consisted of stereotactic aspiration or excision; this study does not mention if EVD was eventually necessary, or if there were complications in terms of hydrocephalus [1].

\section{Treatment modalities of pyocephalus}

Ventricular drainage may be performed on the basis of the patient's clinical state, imaging findings and the response to routine antibiotic therapy. Drainage was not indicated in one patient in good clinical condition in which pyocephalus occurred three weeks after treatment for purulent meningitis, accompanied by severe headaches and fever and the recurrence of meningeal irritation. In the remaining patients, drainage was required for a possible unilateral foramen of Monro obstruction causing hydrocephalus. Black et al described a similar experience in the treatment of a ruptured thalamic abscess, although with complications arising five weeks after the commencement of treatment [11]. Brewer et al recommended drainage as a routine therapy in a paper published in 1975 [12]. The intraventricular administration of vancomycin has been presented as a successful treatment for pyocephalus in a number of previous case studies $[6,13,14]$ although this approach has been unsuccessful in other cases involving gentamicin [15]. Two patients died early on in our study from ongoing sepsis and multiple organ failure. The cause of death was not hydrocephalus and even prompt and aggressive antibiotic therapy was ineffective.

While we believe this approach remains the most appropriate in such cases, it does mean that improving patient outcome will, for the time being, continue to be problematic. The appropriate surgical technique depends on the localisation and the character of the findings. All of these patients were culture-positive, therefore we can assume active inflammation and the possible inefficacy of antibiotic therapy. Furthermore, the permeation of antibiotics into a developed, encapsulated abscess remains debatable and the therapeutic effect impossible to determine directly. Only cefotaxime, ceftazidime, imipenen and metronidazole have been proven to penetrate the abscess capsule in vivo [16-19]. A rare minimally-invasive approach is presented by Nishizaki et al, where the patient successfully underwent evacuation of the intraventricular abscess using a neuro-endoscope. The material was collected for bacterial culture, resulting in gentamicin sulphate being administered twice daily for nine days in combination with intravenous cefotaxime sodium for 14 days. The patient made a full recovery [20].

Vancomycin is the subject of most research in the intraventricular administration of antibiotics. It is considered to be safe in a wide range of doses, from $0.075-50 \mathrm{mg}$ per day. $5-20 \mathrm{mg}$ is the most frequently recommended daily dose to 
achieve a sterile fluid. Only a few instances of side effects have been noted, such as leukocytosis [21] and severe headaches when administered via an Ommaya reservoir [22]. The pharmacokinetics of vancomycin in the ventricular system are unclear, relying as they do on several possible population models, with a range of complicating factors including severe infection, obstruction of CSF flow in hydrocephalus or the presence of drainage [5].

\section{Laboratory findings}

Elevation of inflammation markers in abscesses tends to be unspecific and does not reflect the severity of the illness. Infections in isolated compartments such as the CNS as protected by the blood-brain barrier induce only minimal or slight system changes that can be detected in serum, as reflected in our findings - physiological CRP levels were found in $44.1 \%$ of our study patients. Helweg-Lareson et al conducted a retrospective study of 102 patients and found that $26 \%$ of the cohort had low CRP $(<20 \mathrm{mg} / \mathrm{l})$ and that $49 \%$ were without leukocytosis. CRP levels were not found to influence outcome [23]. Similarly, Ko et al, in a retrospective study, found initial CRP values to be normal in 39\% of the study group [24]. We determined statistically higher CRP among patients with pyocephalus on the day of surgery. However, the current lack of similar published studies means it is not possible to corroborate these findings.

Severe ventriculitis resulting in sepsis, being the system's reaction to insult, elevates acute inflammatory parameters in serum, as consistent with our findings. CRP cannot therefore be taken to be a reliable indicator of a successfully treated infection, and should only be used to supplement imaging and clinical findings in the decision to end antibiotic therapy.

\section{Localisation}

Regarding the supratentorial and infratentorial regions, brain abscesses occur overwhelmingly more frequently in the former. We found abscesses to be most commonly localised in the frontal or frontoparietal lobe, as consistent with other studies $[1,7,25]$. In a cohort of 51 patients, Cavusoglu et al demonstrated its typical occurrence in the temporoparietal lobe [26].

\section{Seizures}

Epileptic seizures in the acute phase are relatively common, occurring in $13-50 \%$ of patients $[1,7,9,27-30] .30-50 \%$ of patients are at risk of late seizures or chronic epilepsy [27-31]. In a three-year study, Buonaguro et al described late seizures in $7 / 29$ children (24\%) [32]. In our study, there was just one patient who experienced repeated seizures requiring combination antiepileptics after six months. The remaining initially symptomatic patients were prescribed anticonvulsants until the conclusion of antibiotic therapy and for a maximum of three months. Abscess localisation in the frontoparietal lobe is a possible risk factor [33], although this could not be confirmed in our study.
It is also necessary to consider the role of medication in inducing seizures: Martin-Canal et al documented a significantly higher incidence of convulsions in patients treated with imipenem compared to meropenem, and recommended this and carbapenem in such cases. The pharmacokinetic and microbial effect is thought to be broadly similar if not identical. Additionally, no seizures were noted in a group administered only metronidazole and cephalosporins [34]. In children, the administration of anticonvulsants should be individualised on the basis of EEG monitoring [30]. Most authors recommend the prophylactic prescription of medication for three months [35]. One possible weakness of the study is the six-month follow up period, given the potential for the late onset or recurrence of seizures, especially in children. At least two years of follow up is required to properly evaluate this complication.

\section{Conclusion}

Although its prognostic value remains uncertain, we do know that pyocephalus will continue to be a serious complication in the treatment of brain abscesses, initially requiring prompt and aggressive antibiotic therapy supplemented by surgical treatment of lesions. We find EVD to be an appropriate procedure for the aspiration of purulent material, and useful for both the prevention of acute hydrocephalus and the possibility of directly administering antibiotics. We were unable to identify any risk factors associated with the development of this complication. These patients have statistically higher CRP on the day of operation. The occurrence of epileptic seizures in the acute phase of brain abscess treatment is a poor prognostic factor.

\section{Conflict of interest: none}

Funding: No funding was received for this research. Informed consent: Informed consent was obtained from all individual participants included in the study.

\section{References}

1. Lee TH, Chang WN, Su TM, et al. Clinical features and predictive factors of intraventricular rupture in patients who have bacterial brain abscesses. J Neurol Neurosurg Psychiatry. 2007; 78(3): 303-309, doi: 10.1136/jnnp.2006.097808, indexed in Pubmed: 17012340.

2. Ferré $C$, Ariza J, Viladrich PF, et al. Brain abscess rupturing into the ventricles or subarachnoid space. Am J Med. 1999; 106(2): 254-257, indexed in Pubmed: 10230756.

3. Takeshita M, Kawamata T, Izawa M, et al. Prodromal signs and clinical factors influencing outcome in patients with intraventricular rupture of purulent brain abscess. Neurosurgery. 2001; 48(2): 310-6; discussion 316, indexed in Pubmed: 11220373.

4. Yang SY, Zhao CS. Review of 140 patients with brain abscess. Surg Neurol. 1993; 39(4): 290-296, indexed in Pubmed: 8488448.

5. Ng K, Mabasa VH, Chow I, et al. Systematic review of efficacy, pharmacokinetics, and administration of intraventricular vancomycin in 
adults. Neurocrit Care. 2014; 20(1): 158-171, doi: 10.1007/s12028012-9784-z, indexed in Pubmed: 23090839.

6. Doan N, Nguyen Ha, Luyuan Li, et al. Good Outcomes with the Intraventricular Vancomycin Therapy in a Patient with Ruptured Brain Abscesses. Asian J Neurosurg. 2018; 13(2): 396-399, doi: 10.4103/17935482.185065, indexed in Pubmed: 29682042.

7. Xiao F, Tseng MY, Teng $\sqcup$, et al. Brain abscess: clinical experience and analysis of prognostic factors. Surg Neurol. 2005; 63(5): 442-9; discussion 449, doi: 10.1016/j.surneu.2004.08.093, indexed in Pubmed: 15883068.

8. Amornpojnimman T, Korathanakhun P. Predictors of clinical outcomes among patients with brain abscess in Thailand. J Clin Neurosci. 2018; 53: 135-139, doi: 10.1016/j.jocn.2018.04.059, indexed in Pubmed: 29716805.

9. Kao PT, Tseng HK, Liu CP, et al. Brain abscess: clinical analysis of 53 cases. J Microbiol Immunol Infect. 2003; 36(2): 129-136, indexed in Pubmed: 12886965.

10. Tunthanathip T, Kanjanapradit K, Sae-Heng S, et al. Predictive factors of the outcome and intraventricular rupture of brain abscess J Med Assoc Thai. 2015; 98: 170-180.

11. Black PM, Levine BW, Picard EH, et al. Asymmetrical hydrocephalus following ventriculitis from rupture of a thalamic abscess. Surg Neurol. 1983; 19(6): 524-527, indexed in Pubmed: 6857481.

12. Brewer NS, MacCarty CS, Wellman WE. Brain abscess: a review of recent experience. Ann Intern Med. 1975; 82(4): 571-576, indexed in Pubmed: 1168037.

13. Isono M, Wakabayashi Y, Nakano T, et al. Treatment of brain abscess associated with ventricular rupture-three case reports. Neurol Med Chir (Tokyo). 1997; 37(8): 630-636, indexed in Pubmed: 9301202.

14. Zeidman SM, Geisler FH, Olivi A. Intraventricular rupture of a purulent brain abscess: case report. Neurosurgery. 1995; 36(1): 189-93; discussion 193, indexed in Pubmed: 7708158.

15. Maeda K, Sanada M, Kawai $H$, et al. Pyogenic ventriculitis with ruptured brain abscess. Intern Med. 2006; 45(13): 835-836, indexed in Pubmed: 16880712.

16. Sjölin J, Eriksson N, Arneborn P, et al. Penetration of cefotaxime and desacetylcefotaxime into brain abscesses in humans. Antimicrob Agents Chemother. 1991; 35(12): 2606-2610, indexed in Pubmed: 1810195.

17. Green HT, O'Donoghue MA, Shaw MD, et al. Penetration of ceftazidime into intracranial abscess. J Antimicrob Chemother. 1989; 24(3): 431-436, indexed in Pubmed: 2681122.

18. Asensi V, Cartón JA, Maradona JA, et al. Therapy of brain abscess with imipenem--a safe therapeutic choice? J Antimicrob Chemother. 1996; 37(1): 200-203, indexed in Pubmed: 8647769.

19. Ingham HR, Slekon JB, Roxby CM. Bacteriological study of otogenic cerebral abscesses: chemotherapeutic role of metronidazole. Br Med J. 1977; 2(6093): 991-993, indexed in Pubmed: 922400.

20. Nishizaki T, Ikeda N, Nakano S, et al. Successful neuroendoscopic treatment of intraventricular brain abscess rupture. Clin Pract. 2011; 1(3): e52, doi: 10.4081/cp.2011.e52, indexed in Pubmed: 24765313.
21. Hirsch BE, Amodio M, Einzig Al, et al. Instillation of vancomycin into a cerebrospinal fluid reservoir to clear infection: pharmacokinetic considerations. J Infect Dis. 1991; 163(1): 197-200, indexed in Pubmed: 1984469.

22. Sutherland GE, Palitang EG, Marr JJ, et al. Sterilization of Ommaya reservoir by instillation of vancomycin. Am J Med. 1981; 71(6): 10681070, indexed in Pubmed: 7315852.

23. Helweg-Larsen J, Astradsson A, Richhall $\mathrm{H}$, et al. Pyogenic brain abscess, a 15 year survey. BMC Infect Dis. 2012; 12: 332, doi: 10.1186/1471-2334-12-332, indexed in Pubmed: 23193986.

24. Ko SJ, Park KJ, Park DH, et al. Risk factors associated with poor outcomes in patients with brain abscesses. J Korean Neurosurg Soc. 2014; 56(1): 34-41, doi: 10.3340/jkns.2014.56.1.34, indexed in Pubmed: 25289123.

25. Zhang C, Hu L, Wu X, et al. A retrospective study on the aetiology, management, and outcome of brain abscess in an 11-year, single-centre study from China. BMC Infect Dis. 2014; 14: 311, doi: 10.1186/14712334-14-311, indexed in Pubmed: 24903315.

26. Cavuşoglu H, Kaya RA, Türkmenoglu ON, et al. Brain abscess: analysis of results in a series of 51 patients with a combined surgical and medical approach during an 11-year period. Neurosurg Focus. 2008; 24(6): E9, doi: 10.3171/FOC/2008/24/6/E9, indexed in Pubmed: 18518754.

27. Carpenter J, Stapleton S, Holliman R. Retrospective analysis of 49 cases of brain abscess and review of the literature. Eur J Clin Microbiol Infect Dis. 2007; 26(1): 1-11, doi: 10.1007/s10096-006-0236-6, indexed in Pubmed: 17180609.

28. Goodkin HP, Harper MB, Pomeroy SL. Intracerebral abscess in children: historical trends at Children's Hospital Boston. Pediatrics. 2004; 113(6): 1765-1770, indexed in Pubmed: 15173504.

29. Lee CG, Kang SH, Kim YJ, et al. Brain abscess in Korean children: A 15year single center study. Korean J Pediatr. 2010; 53(5): 648-652, doi: 10.3345/kjp.2010.53.5.648, indexed in Pubmed: 21189932.

30. Sáez-Llorens X, Nieto-Guevara J. Brain abscess. Handb Clin Neurol. 2013; 112: 1127-1134, doi: 10.1016/B978-0-444-52910-7.000325, indexed in Pubmed: 23622320.

31. Osenbach RK, Loftus CM. Diagnosis and management of brain abscess. Neurosurg Clin N Am. 1992; 3(2): 403-420, indexed in Pubmed: 1633468.

32. Buonaguro A, Colangelo $M$, Daniele $B$, et al. Neurological and behavioral sequelae in children operated on for brain abscess. Childs Nerv Syst. 1989; 5(3): 153-155, indexed in Pubmed: 2758427.

33. Chuang MJ, Chang WN, Chang HW, et al. Predictors and long-term outcome of seizures after bacterial brain abscess. J Neurol Neurosurg Psychiatry. 2010; 81(8): 913-917, doi: 10.1136/jnnp.2009.195073, indexed in Pubmed: 20682720.

34. Martin-Canal G, Saavedra A, Asensi JM, et al. Meropenem monotherapy is as effective as and safer than imipenem to treat brain abscesses. Int J Antimicrob Agents. 2010; 35(3): 301-304, doi: 10.1016/j.ijantimicag.2009.11.012, indexed in Pubmed: 20045289.

35. Cochrane DD. Consultation with the specialist. Brain abscess. Pediatr Rev. 1999; 20(6): 209-215, indexed in Pubmed: 10352044. 